\title{
Thermal Profiles of High-voltage Capacitor Units
}

\author{
Calum J. Mackinnon \\ Department of Electronic and Electrical Engineering \\ University of Strathclyde \\ Glasgow, Scotland \\ Calum.Mackinnon@Strath.ac.uk
}

\author{
Brian G. Stewart \\ Department of Electronic and Electrical Engineering \\ University of Strathclyde \\ Glasgow, Scotland \\ Brian.Stewart.100@Strath.ac.uk
}

\begin{abstract}
High voltage capacitors are useful in power factor correction, harmonic filtering, energy storage and voltage support for modern electrical power systems. Even modest failure rates can be compounded by large numbers of assets deployed in high capacity installations, and as fuses are positioned internally or omitted in pursuit of more efficient capacitor designs, it becomes increasingly challenging to identify the location of a faulted element within a module. This paper investigates detectability of an internal heat source from distributions of temperature on a capacitor unit's outer housing. A model of a high voltage capacitor module is presented and is used to simulate the propagation of thermal energy from a heat source sited at select positions within a unit to a steel enclosure. Resultant heat distributions are shown to be readily influenced by foil arrangement and heat source location within a capacitor, since foils both direct and contain heat.
\end{abstract}

Keywords-Capacitors, Fault Detection, Partial Discharge

\section{INTRODUCTION}

Power electronic conversion allows a modern alternative to conventional power system reinforcement: network capacity can be increased through power factor correction, high voltage direct current links, series compensation, and connection of renewable sources dispersed across a network in terms of both connection voltage and location. As such technologies bear responsibility for greater network capacities, they become more critical to network operation and as a constituent component of power electronic interfaces and standalone power factor correction, shunt capacitor banks correspondingly become "increasingly important" for reliable power systems [1], across voltage levels.

Capacitor units are generally considered a relatively reliable part of the wider system, and that banks comprise modular units aids partial replacement. However, even modest failure rates could become cumbersome when scaled to match the number of components employed for high-capacity network applications, and as units are unrepairable [1], early detection and tracking for developing faults and asset degradation is preferable. Accordingly, this paper explores how the location of a point heat source influences heat profiles of a module's external housing, by employing a simulated model of a high voltage capacitor unit as illustrated by Figure 1, which highlights internal elements.

Section II introduces technical background for capacitor units, particularly in terms of their design and construction. Section III then introduces a simulation model developed in COMSOL Multiphysics, with which simulation studies produce the preliminary results presented in Section IV. These results are discussed in Section V and conclusions are drawn in Section VI.

\section{BACKGROUND}

Individual capacitor modules are connected in banks, for which each side of a bridge arrangement is expected to remain balanced with its complement, save a sufficient imbalance from sudden or gradual degradation trips a protection relay. This traditional approach is unable to discern concurrent degradation on parallel branches, where a bridge can remain balanced [2].

Capacitor elements are constructed from discrete layers of aluminium foil (to which tabs are connected) and a dielectric, which together are rolled into a compact form [3]. Foil tabs on each terminal are then used to connect these elements, first in parallel into groups termed 'series elements' or "series sections" [2], which then are connected in series into a stack. A unit's voltage rating and capacity for reactive power support are respectively determined by the total capacitance connected in series and in parallel in this manner, and which vary by design.

Resilience to faults can also vary by design, as fuses can be configured externally, internally, or omitted (in place of 'selfhealing' options wherein fault energy vaporises an area of foil). Internally fused and self-healing capacitor modules allow a series section or even only a small element area to be protected for the remainder of the module to stay in service, although these approaches can be less efficient during normal operation. A technique presented in [4] enables a series element failure to be detected and tracked to one leg of a capacitor bank bridge arrangement. Otherwise, it is difficult to identify a specific unit of an operational capacitor bank as the location of a subtle fault.

Prior to fuse operation, an insulation defect can invite partial discharge to deteriorate a dielectric between terminals. Partial discharges act as heat sources and can occur anywhere within the dielectric of a module but are more probable under high electrical stress. Where insulation degrades to a point of failure between terminals of a capacitor element, a short-circuit trips a fuse. In this eventuality, terminals are short-circuited, and the overall value of capacitance is therefore reduced. In turn, increased reactance attracts greater voltages which consequently are shared by the fewer elements remaining in the capacitor bank. Left unchecked, such scenarios increase voltage stress on remaining elements, which thereby provoke further series element failures and increase susceptibility of the whole bank to cascading element failure [4]. Foil tab geometries can aggravate electrical stresses and these points of connection are additionally prone to resistive heating as current is conveyed between elements, even where such connections are electrically sound. 
For every 8 to $10{ }^{\circ} \mathrm{C}$ increase in ambient temperature, the expected useful life of a capacitor unit halves [5], [2]. This applies to capacitor units operating in a 40 to $65{ }^{\circ} \mathrm{C}$ operating range [6], and may vary according to internal design, unit size, surface and environmental factors. Capacitor module dielectrics generate heat under normal conditions: [7] estimates the temperature rise which occurs throughout the dielectric volume of a capacitor unit, and shows it relates to the value of $\tan \delta$; and [8] considers the transfer of heat within individual self-healing capacitor elements used in pulsed power applications. By generating heat, these dielectric losses could mask any signal from a heat source of particular interest within a capacitor unit.

An ability to detect incipient faults and series element failure or locate them within a bank of capacitor units could allow degradation to be monitored, would reveal faults on parallel arms of a bridge arrangement, and could inform operation and maintenance decisions, principally to pick units for replacement.

\section{Simulation STUdiES}

A configurable capacitor unit model (Figure 1) has been developed in COMSOL Multiphysics ${ }^{\circledR}$ such that it can be set to simulate foils stacked according to either an $\mathrm{X}, \mathrm{Y}$, or $\mathrm{Z}$ axis. It is configured with 3 groups of 6 capacitive elements comprising discrete aluminium foils, each wound 4 turns with dielectric of relative permittivity 3.9 to mimic mineral oil and paper layers. Each element is a series of flat foil surfaces and turns which connect them. In COMSOL, a boundary condition to emulate heat transfer in solids specifies thermal insulation for module materials (including element foils); a $10.45 \mathrm{Wm}^{-2} \mathrm{~K}^{-1}$ heat flux transfer coefficient allows unit housing to cool in a room temperature environment; a $50 \mathrm{Wm}^{-3}$ heat is applied throughout the dielectric to emulate effects of dielectric losses, and a $0.2 \mathrm{~W}$ point heat source is specified at each of a set of distinct locations.

This capacitor model is simplistic but is intended primarily to illustrate the role of heat in forming profiles on outer housing. Cut lines are specified on each face of the module housing to allow heat profiles to be graphed and are illustrated in Figure 2, which names housing faces and cut lines relative to model axes. Another cut line (dotted, in Figure 2) centrally bisects all foils.

To avoid repetition, a module with elements stacked along the $\mathrm{X}$ axis (vertically, as a module would typically sit on a rack, and as shown in Figure 1) is simulated to explore the propagation of heat from different locations within the unit. Three such point heat source locations are tested: a foil tab connection; a second-from-last foil in an element roll; and a centremost foil edge, where all apply to a central $\left(9^{\text {th }}\right)$ element. Rather than correspond to specific types of fault, these scenarios illustrate how thermal profiles can change with source position.

\section{RESULTS}

Effects of a point heat source contained within a central capacitive element on a module simulated with foils stacked along the X, Y, and Z axes are shown in Figure 3, Figure 4, and Figure 5 respectively. Figure 6, Figure 7, Figure 8 each include four graphs, which respectively show measurements across cut lines for a $0.2 \mathrm{~W}$ heat source on an edge of: an innermost foil; a penultimate foil; and a tab connector, of an X-aligned foil unit.

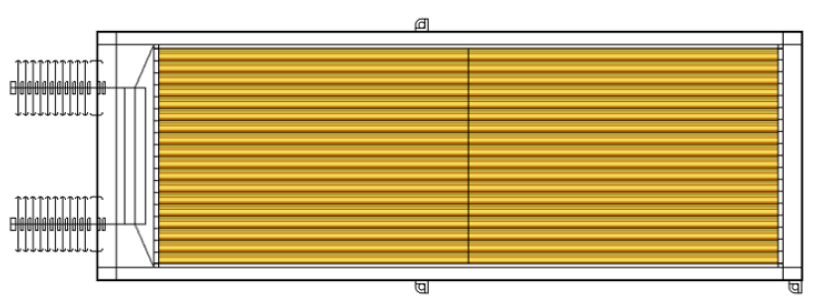

Fig. 1. A cross-section of the model geometry

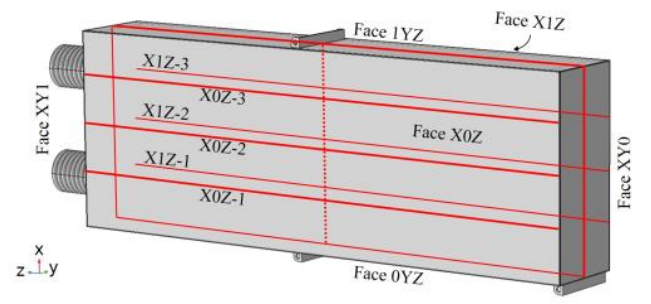

Fig. 2. An illustration of cut lines specified to generate heat profiles

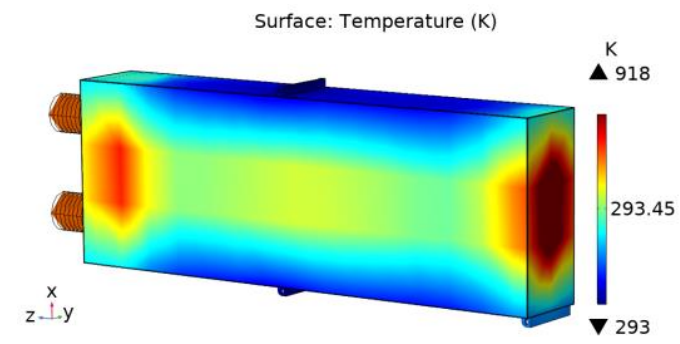

Fig. 3. Heat on a module with $\mathrm{X}$-aligned foils

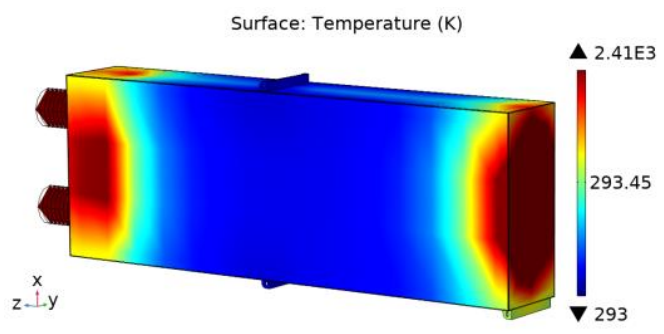

Fig. 4. Heat on a module with Y-aligned foils

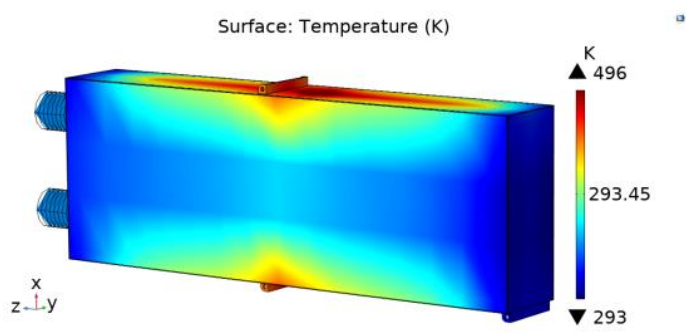

Fig. 5. Heat on a module with Z-aligned foils 

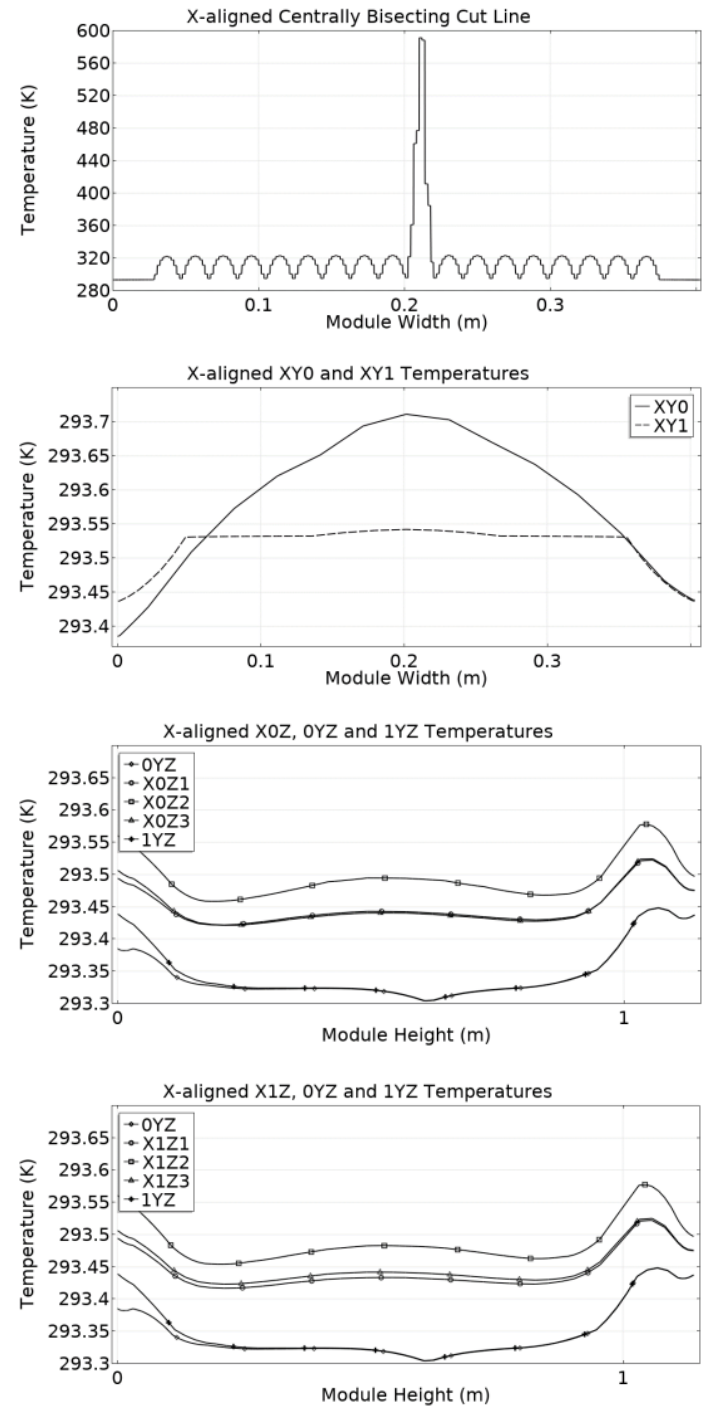

Fig. 6. Cut-line thermal profiles for heat on an innermost element foil
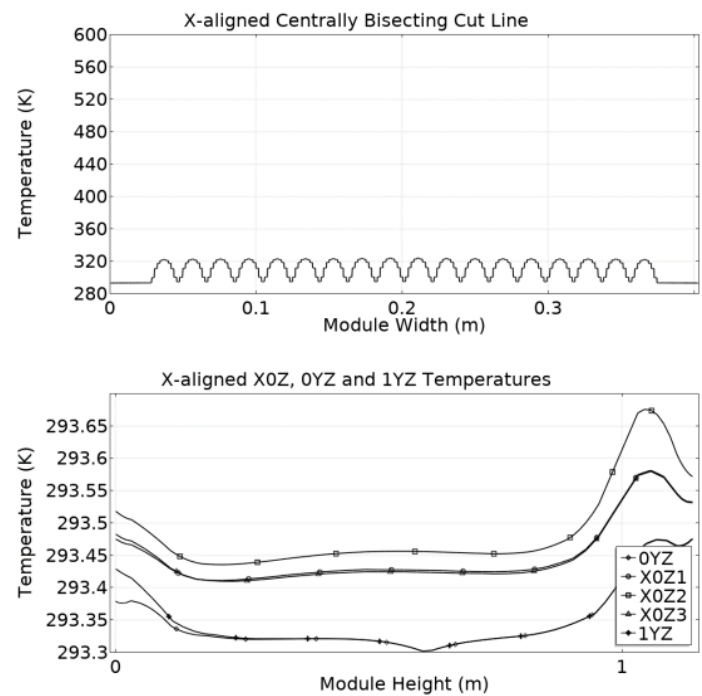
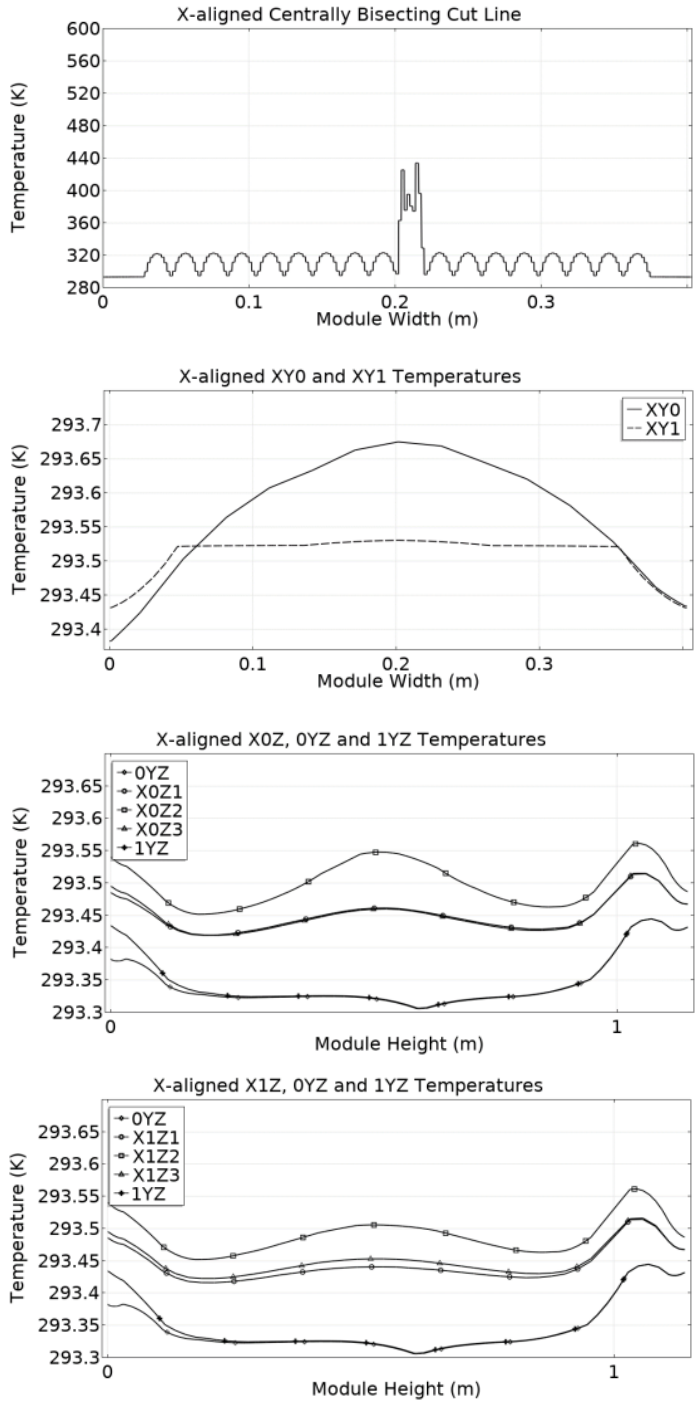

Fig. 7. Cut-line thermal profiles for heat on a penultimate element foil
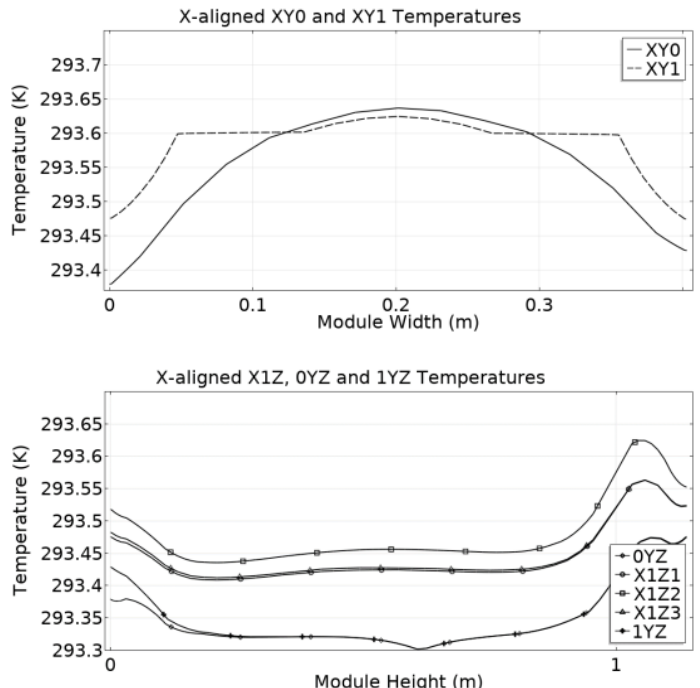

Fig. 8. Cut-line thermal profiles for heat on a foil tab connector 


\section{DISCUSSION}

Thermally conductive aluminium foils predominantly direct thermal energy according to their alignment, toward whichever faces of a capacitor module are parallel to neither flat foil surfaces nor turns made as foils fold into elements. Figure 3, Figure 4, and Figure 5 reveal that foil alignment significantly alters heat propagation characteristics of a similar capacitor unit from a similar position on a centremost foil of a central $\left(9^{\text {th }}\right)$ element, but these distinct cases share commonality: in each, two faces expend a large amount of heat; two are intermediately warm; and two remain comparatively cool. Warmest faces are those perpendicular to both foils' planar surfaces and turns; lukewarm faces are perpendicular to planar foil surfaces but parallel with turns; and coolest faces are parallel with foil faces.

Comparing the topmost line graphs of Figure 6 (a centre foil) and Figure 7 (a penultimate foil), the maximum temperature in the first case is more than twice that of the second. Temperature increases on the 'X0Z' and 'X1Z' faces are also less pronounced in Figure 6: for heat at an innermost foil, energy is more readily contained by foils such that it cannot as easily spread throughout an element, as indicated by a pronounced spike in Figure 7, and is less able to reach the module container. Thus, thermally insulating aluminium foils contain thermal energy in an element.

If a heat source occurs elsewhere within an element, then the direction in which foils are rolled allows thermal energy to more readily reach one intermediately heated housing face than it does the opposite face. As such, two faces of a unit container affected by a point heat source will be intermediately heated to differing extents (as seen by different temperature increases in the thirdand fourth-line graphs of Figure 7). A temperature increase on one intermediately warm face relative to the other suggests an approximate position of a heat source within an element, given prior knowledge of turn direction. Were manufacturers to alternate the foil fold direction of adjacent elements, it could aid accuracy of location estimates which employ such information.

Compared with the scenarios in Figure 6 and in Figure 7: the first graph of Figure 8 shows no distinct spike of temperature, suggesting elements remain comparatively cool; a markedly higher temperature is found across face ' $\mathrm{XY} 1$ ' of the housing (where bushings are); and a correspondingly high temperature results in a pronounced signal toward the right of ' $\mathrm{XOZ}$ ' and 'X1Z' line graphs. Hence, a heat source on a foil tab increases temperature at only one end of the capacitor: any temperature rise is asymmetrical; and no two opposing faces are heated.

It could be possible to locate a source of heat to: a capacitive element; a dielectric layer within the element; and to a location on the foil layer. Correspondingly, heat-emitting faults such as partial discharge could be detected (and to some extent located) within a capacitor unit should thermal profiles be measurable, such as with sensitive infra-red imaging, distributed temperature sensors, or heat-sensitive stickers on a unit housing. Localised dielectric heating can affect relative permittivity, and thereby alter the capacitance of an element within the unit. As such, any means of detection could also be corroborated electrically.

Relative to real, commercially available capacitor modules, this preliminary steady-state study makes simplifications: dielectric losses and environmental influences are omitted; a unit model is a simplistic approximation; and time taken for heat to propagate to the outermost enclosure is ignored but matters for intermittent heat sources. Instead, commercially available units vary in their: size and structure; internal design; dielectric materials; environment; and electrical loading. Results of this study are accordingly subject to more nuanced and realistic observations which require validation in a laboratory setting.

Future work may consider the discernibility of a heat source from measurements which can be affected by the presence of temperature sources within a capacitor module but also ambient sources of heat and those intrinsic to the role of an asset within an electrical circuit, since electrical loading and the incidence of harmonics will both affect the temperature of healthy capacitors.

\section{CONCLUSIONS}

Thermal profiles across a unit housing are predominantly influenced by internal foil arrangement. Incipient faults such as partial discharge could be detected thermally, but any preferable measurement locations or data interpretation would rely on knowledge of internal designs. Hence, manufacturers may be best positioned to detect and locate unit faults toward reliability centred maintenance, given this prerequisite knowledge.

As heat propagates according to foil alignment, an element containing a heat source can best be inferred from a temperature distribution on the warmest housing faces; a foil surface (depth) within the element can be approximated from temperatures of intermediately heated faces, relative to those of the warmest faces and to one another; and heat sources outwith foil elements can be recognised by overall asymmetric thermal profiles.

While this simplistic study omits realistic sources of thermal noise, it illustrates principles by which location of a subtle fault such as partial discharge can be inferred. Such inference would use knowledge of internal module design and an understanding of how thermal energy propagates to encode the location of its origin in thermal profiles of high-voltage capacitor units.

\section{REFERENCES}

[1] M. A. El-hadidy, H. Helmi, "Failure analysis of medium voltage capacitor banks: the Egyptian experience", CIRED 2009.

[2] M. Ennis, D. J. Meisner, M. Thakur, "Innovative protection schemes for $\mathrm{H}$ configuration fuseless grounded shunt capacitor banks", $65^{\text {th }}$ Annual Conference for Protective Relay Engineers, pp. 449-458, 2012.

[3] W. J. Sarjeant, J. Zirnheld, F. W. MacDougall, "Capacitors", IEEE Transactions on Plasma Science, Vol. 26, No. 5, October 1998.

[4] H. Jouybari-Moghaddam, T. S. Sidhu, M. R. Dadash Zadeh, P. P. Parikh, "Enhanced fault-location scheme for double wye shunt capacitor banks", IEEE Transactions on Power Delivery, Vol. 32, No. 4, August 2017.

[5] M. G. Kong, Y. P. Lee, "Electrically induced heat dissipation in metallized film capacitors", IEEE Transactions on Dielectrics and Electrical Insulation, Vol. 11, No. 6, pp. 1007-1013, December 2004.

[6] W. J. Sarjeant, F. W. MacDougall, D. W. Larson, I. Kohlberg, "Energy storage capacitors: aging, and diagnostic approaches for life validation", IEEE Transactions on Magnetics, Vol. 33, No. 1, January 1997.

[7] S. H. Lee, B. Y. Lee, H. K. Kim, H. G. Kim, "Local heat source approximation technique for predicting temperature rise in power capacitors”, IEEE Trans. Magnetics, Vol. 45, No. 3, March 2009.

[8] Z. Li, H. Li, X. Huang, H. Li, W. Want, B. Wang, F. Lin, Q. Zhang, "Temperature rise of metallized film capacitors in repetitive pulse applications", IEEE Trans. Plasma Science, Vol. 43, No. 6, June 2015. 